\section{Corinne Invernizzi}

\section{Essenzielle und nicht}

essenzielle Fette [1-3]

Fettsäuren (FS) können nach der Zahl der Doppelbindungen in gesättigte FS, einfach ungesättigte FS und mehrfach ungesättigte FS eingeteilt werden. Anhand ihrer Kettenlänge lassen sie sich weiter in niedere ( $\leq 7 \mathrm{C}$-Atome), mittlere (8-12 C-Atome) und höhere (> 12 C-Atome) FS unterteilen. Die vom Säugetier stammenden gesättigten FS besitzen keine Doppelbindung und gelten als nicht essenziell, da der menschliche Organismus in der Lage ist, diese selbst zu syntheti-

\title{
Die Rolle von Nachtkerzensamenöl bei chronisch-entzündlichen Prozessen begleitet von einem Gamma-Linolensäure-Mangel
}

sieren. Ebenfalls zu den nicht essenziellen FS zählen die zum Beispiel in Rapsöl und Olivenöl enthaltenen Omega-9-FS. Sie sind einfach ungesättigte FS und verfügen lediglich über eine Doppelbindung.

Für den menschlichen Organismus essenziell sind dagegen die mehrfach ungesättigten FS, wie die Omega-3FS, die vorwiegend im Fischöl vorkommen und die in vielen pflanzlichen Ölen enthaltenen Omega-6-FS. Eine für die Medizin, insbesondere in der Dermatologie, relevante Omega6-FS ist die Gamma-Linolensäure (GLA).

\section{Zentrale Rolle der Gamma- Linolensäure bei der atopischen Dermatitis $[4,5]$}

Bei der atopischen Dermatitis (Neurodermitis atopica, atopisches Ekzem, chronisch-konstitutionelles Ekzem) können im Serum mitunter erhöhte Linolsäurewerte sowie erniedrigte GLA-Werte und Dihomo-GammaLinolensäure (DGLA) gemessen werden. Dieses Phänomen scheint auf einem gestörten Fettsäurestoffwechsel (Abb. 1) der Haut zu beruhen, dessen zentrales Problem seinerseits auf einem Enzymmangel der Delta-6-De-
Abb. 1. Fettsäurestoffwechsel der Omega-6-FS (modifiziert nach [22]).

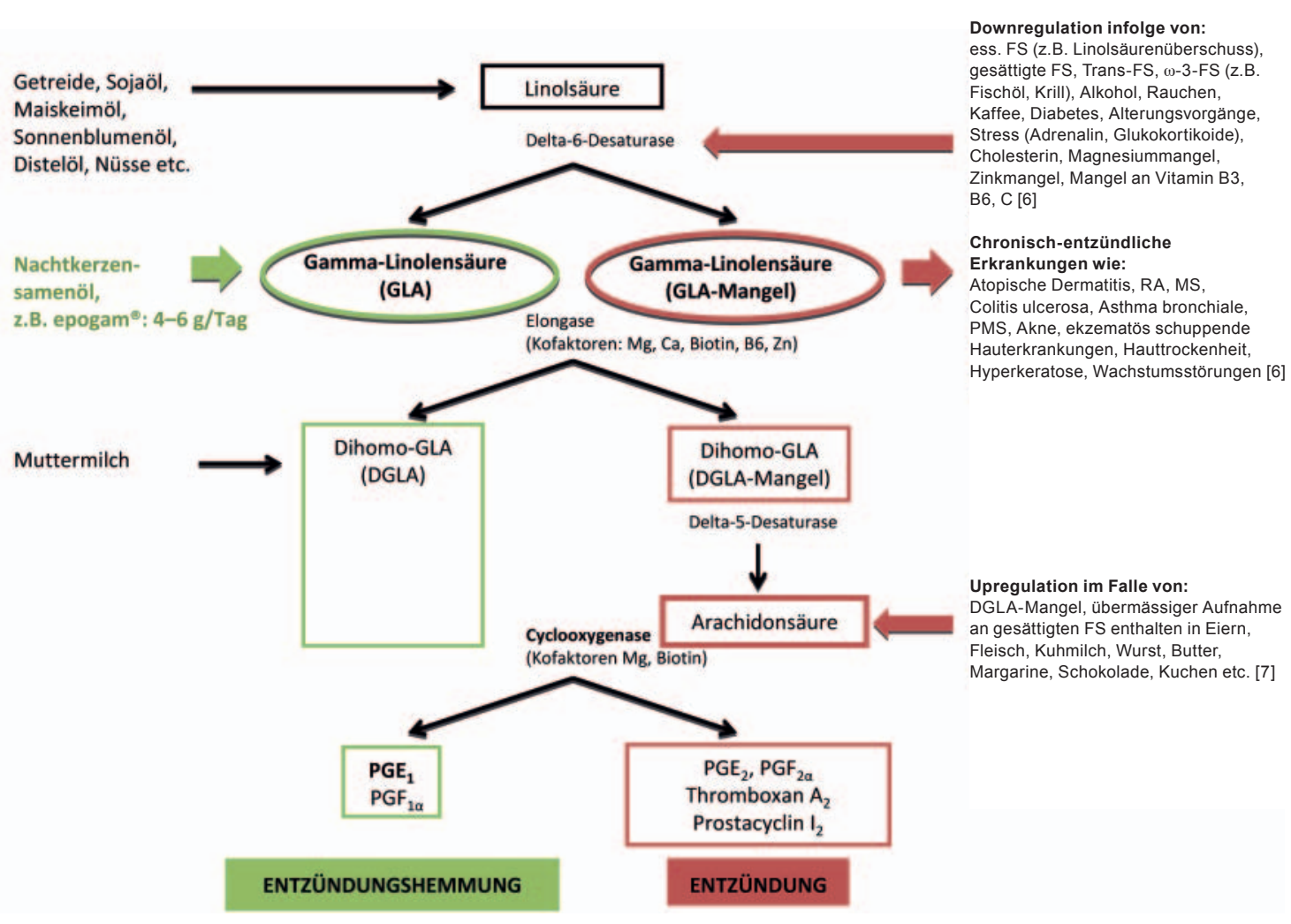

\section{KARGER}

Fax +49 7614520714 Information@Karger.de www.karger.com
(๑) 2011 S. Karger GmbH, Freiburg 
saturase basiert. Durch die mangelhafte Synthese der GLA und deren Umwandlung in die DGLA kommt es zur Gleichgewichtsverschiebung zugunsten der stark entzündungsund thrombosefördernden Arachidonsäure, die vorwiegend aus tierischen Fetten und Omega-6-FS (Linolsäure) synthetisiert wird. Aus DGLA werden unter anderem entzündungshemmende, blutdrucksenkende und vasodilatatorische Prostaglandine der Serie I gebildet, mit dem besonders für Atopiker bedeutenden Vertreter $\mathrm{PGE}_{1}$. Arachidonsäure hingegen ist Ausgangssubstanz zahlreicher inflammatorischer Entzündungsmediatoren, wie Prostagladine, Thromboxane und Prostacycline der Serie II sowie Leukotriene der Serie IV, Lipoxine und Hydroxyfettsäuren. Da sowohl die DGLA als auch die Arachidonsäure um das gleiche Enzym, Cyclooxygenase, konkurrieren, werden bei der atopischen Dermatitis anstelle von $\mathrm{PGE}_{1}$ vermehrt inflammatorische Botenstoffe gebildet, was im Entzündungsprozess von entscheidender Bedeutung ist. Durch den Mangel an $\mathrm{PGE}_{1}$ wird die Freisetzung von Histamin und anderen Mediatoren aus Entzündungszellen, wie Leukozyten, Mastzellen und basophilen Granulozyten, begünstigt und die allergisch-entzündliche Reaktion potenziert. Der Circulus vitiosus wird vermutlich durch Histamin weiter in Gang gehalten, da Histamin immunsuppressive Eigenschaften besitzt und in der Lage ist, die Delta-6-Desaturase weiter $\mathrm{zu}$ hemmen. Auch $\mathrm{PGE}_{1}$ zeigt neben einer antiallergischen und antiinflammatorischen Wirkung eine ausgeprägte immunregulatorische Wirkung. Bedingt durch den $\mathrm{PGE}_{1}$-Mangel kommt es $\mathrm{zu}$ einer Funktionsschwäche der regulatorischen T-Zellen, die auf die mangelhafte T-Zell-Reifung und -Differenzierung zurückgeführt werden kann und so die erhöhte Anfälligkeit für bakterielle Infektionen bei Atopikern erklärt. Weiter kommt es zu einer mangelhaften Kontrolle der B-Lymphozyten, die für die teilweise beobachtete überschiessende $\operatorname{Ig}(\mathrm{Im}$ munglobulin)E-Antwort und die gemessenen erhöhten IgE-Werte im Serum mitverantwortlich sind.

\section{Auslösende Faktoren sowie}

Bedeutung des Gamma-LinolensäureMangels bei chronisch-entzündlichen Erkrankungen [6-8]

Niedrige GLA-Werte im Blut können Hinweise geben auf einen vorliegenden GLA-Mangel, dessen Ursprung mitunter in einer Downregulation der äusserst störanfälligen Delta-6-Desaturase zu suchen ist (Abb. 1). Gesättigte Fettsäuren, Trans-Fettsäuren, Linolsäure-reiche Öle (z.B. Distelöl, Sonnenblumenöl), Omega-3-FS (z.B. Fischöl, Krill, Leinsamenöl), Diabetes, Alkohol, Rauchen, Kaffee, Alterungsvorgänge (z.B. Alterspruritus), Stress (Adrenalin und Glukokortikoide), hohe Cholesterinspiegel, Zinkmangel, Magnesiummangel sowie ein Mangel an Vitamin B3, B6 und $C$ können die Delta-6-Desaturase hemmen. Weiter können tiefe GLAWerte mitunter auch bei chronischentzündlichen Erkrankungen, wie Rheumatoide Arthritis (RA), Multiple Sklerose (MS), Colitis ulcerosa, Asthma bronchiale und prämenstruelles Syndrom (PMS) mit einhergehenden starken Periodenschmerzen und Brustspannen, festgestellt werden. Im Besonderen sollte bei vorliegender atopischer Dermatits, aber auch bei Akne oder ekzematösen, schuppenden Hauterkrankungen an einen eventuell vorliegenden GLAMangel gedacht werden. Bei Kindern können Wachstumsstörungen, Hauttrockenheit, Hautschuppung und Hyperkeratose auf einen GLA-Defizit hinweisen. Bei starkem Verdacht auf eine GLA-Mangellage kann der Fettsäurestatus durch ein Labor abgeklärt werden. Im Falle von patho-

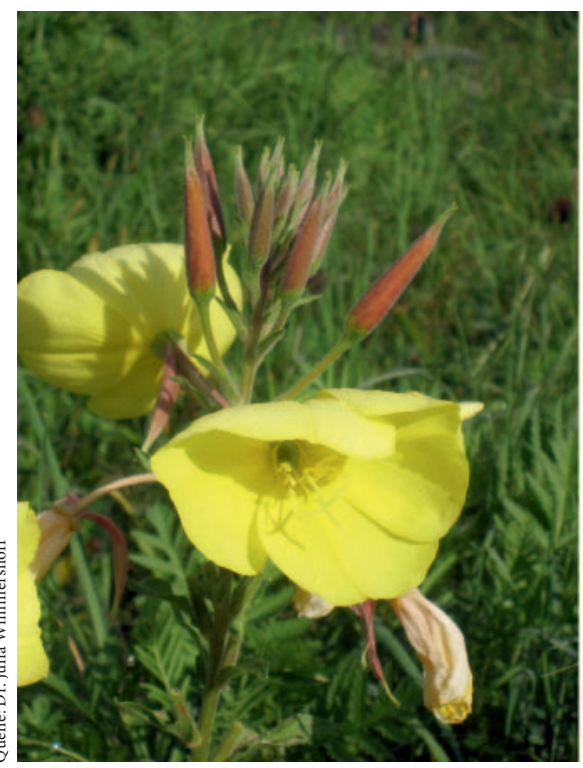

Abb. 2. Nachtkerze (Oenothera biennis L.).

logisch tiefen GLA-Werten ist eine orale Substitutionstherapie (laborkontrolliert) mit Nachtkerzensamenöl (Abb. 2) sinnvoll. An dieser Stelle muss aber darauf hingewiesen werden, dass eine GLA-Substitution zwar wertvoll ist, mit Ausnahme von PMS und bei Menstruationsschmerzen jedoch als alleinige Massnahme häufig nicht ausreicht. Neben weiteren Behandlungsmassnahmen hängt der Behandlungserfolg mitunter auch von der Bereitschaft des Patienten $a b$, auf eine gesunde, ausgewogene Ernährung, die arm an tierischen Fetten bzw. Arachidonsäure ist, umzusteigen. Der tägliche Verbrauch an Arachidonsäure, der für die Eicosanoidbiosynthese (z.B. Prostagladine, Thromboxane, Prostacycline und Leukotriene) benötigt wird, beträgt höchstens 0,001 g pro Tag. Tatsache jedoch ist, dass in den Industrienationen täglich die 200- bis 300-fache Menge davon aufgenommen wird. Im Falle einer Umstellung auf eine vegetarisch orientierte Kost muss deshalb berücksichtigt werden, dass es mehrere Wochen dauert, bis der Körper einen Arachidonsäure-Überschuss verstoffwechselt hat. Der positive Einfluss einer solchen Ernährung auf chronisch-entzündliche Erkran- 
kungen kann deshalb frühestens nach etwa 2 Monaten festgestellt werden.

Therapie eines Gamma-LinolensäureMangels mit hochdosiertem Nachtkerzensamenöl

GLA scheint in den Nahrungsmitteln nicht in allzu grossen Mengen vorzukommen [6]. Eine der wenigen Ausnahmen stellt das Nachtkerzensamenöl dar (Tab. 1). In der Schweiz ist derzeit nur ein Nachtkerzensamenöl (epogam ${ }^{\circledR} 1000$ vegicaps soft $^{\circledR}$; Zeller Medical AG, Romanshorn, Schweiz) kassenzulässig in der Grundversicherung [9]. Die gute Wirksamkeit von Nachtkerzensamenöl konnte bei atopischer Dermatitis, aber auch bei anderen pathologischen Erscheinungen wie PMS in klinischen Studien belegt werden [10-13]. Besonders bei der atopischen Dermatitis hat sich ergänzend zur topischen Basisthera-

Tab. 1. Fettsäurezusammensetzung von Nachtkerzensamenöl [3]

\begin{tabular}{ll}
\hline Fettsäure & Anteil, \% \\
\hline Linolsäure & $65-80$ \\
Gamma-Linolensäure (GLA) & $8-14$ \\
Ölsäure & $6-11$ \\
Palmitinsäure & $7-10$ \\
Stearinsäure & $1,5-3,5$ \\
\hline
\end{tabular}

pie die systemische Langzeitverabreichung von Nachtkerzensamenöl bewährt (Abb. 1). Klinische Studien belegen, dass mit der peroralen Verabreichung von Nachtkerzensamenöl der transdermale Wasserverlust [14] sowie die Entzündungsneigung der Haut gesenkt und eine signifikante Linderung der klinischen Symptome wie Juckreiz (Abb. 3), raue Haut, Ödem und Erythembildung erreicht wird [11]. In der Folge nehmen Intensität und Frequenz der Ekzemintervalle ab, weshalb der Verbrauch an Akutphasen-Therapeutika wie topischen Glukokortikoiden längerfristig reduziert werden kann (Abb. 4) [12]. Um einen maximalen Effekt zu erreichen, wird empfohlen, mit der höchsten Dosierung von $6 \mathrm{~g}(2 \times 3 \mathrm{~g})$ pro Tag $\mathrm{zu}$ beginnen [9]. Die Behandlungsdauer sollte mindestens 3 Monate betragen, da erst innerhalb von einem Zeitraum von 8-12 Wochen mit einer erkennbaren Wirkung gerechnet werden darf [9]. Sobald sich ein Behandlungserfolg zeigt, kann mit der Erhaltungsdosis von $4 \mathrm{~g}(2 \times 2 \mathrm{~g})$ pro Tag fortgefahren werden [9]. In einer Studie konnte zudem gezeigt werden, dass schon nach $4 \mathrm{~h}$ die maximale GLA-Serumkonzentration im Blut erreicht wird, jedoch nach weiteren 8 h die GLA-Werte bereits wieder den
Ausgangswerten entsprechen, was auf eine rasche Metabolisierung der GLA hindeutet [15]. Eine zweimalige Verabreichung von 2-3 g pro Tag erscheint basierend auf diesen Beobachtungen für einen optimalen Behandlungserfolg notwendig zu sein [9].

Bei weiteren Indikationen, wie diabetische Neuropathie und Retinopathie, Klimakterium, RA, Asthma bronchiale, Tumorerkrankungen, ADHS, trockenes Auge, Osteoporose, stoffwechselbedingte Hautekzeme und Hautallergien, Altershaut, kardiovaskuläre Erkrankungen sowie Arterioskleroseprophylaxe, werden die im Öl der Nachtkerze innewohnenden Heilkräfte häufig und gerne propagiert; trotzdem aber legen die meisten zu diesen Indikationen durchgeführten klinischen Studien ein nur vages Zeugnis über die Wirksamkeit des Nachtkerzensamenöls ab [6, 1621]. Ein Grund für die unbefriedigenden Ergebnisse mag darin liegen, dass die Therapiezeiträume vieler Studien unter einer Mindestanwendungsdauer von 3 Monaten lagen und/oder die für einen therapeutischen Effekt notwendige Dosierung von 4-6 g pro Tag nicht eingehalten wurde; diese lag in vielen Fällen weit unterhalb der empfohlenen therapeutischen Dosis im Bereich von 0,5 g pro Tag.
Abb. 3.

Hochsignifikante Juckreizreduktion mit und ohne Substitution von Nachtkerzensamenöl bei atopischer Dermatitis [13].

\begin{abstract}
Abb. 4. Verbrauch topischer Glukokortikoide mit und ohne Substitution von Nachtkerzensamenöl bei atopischer Dermatitis [12].
\end{abstract}
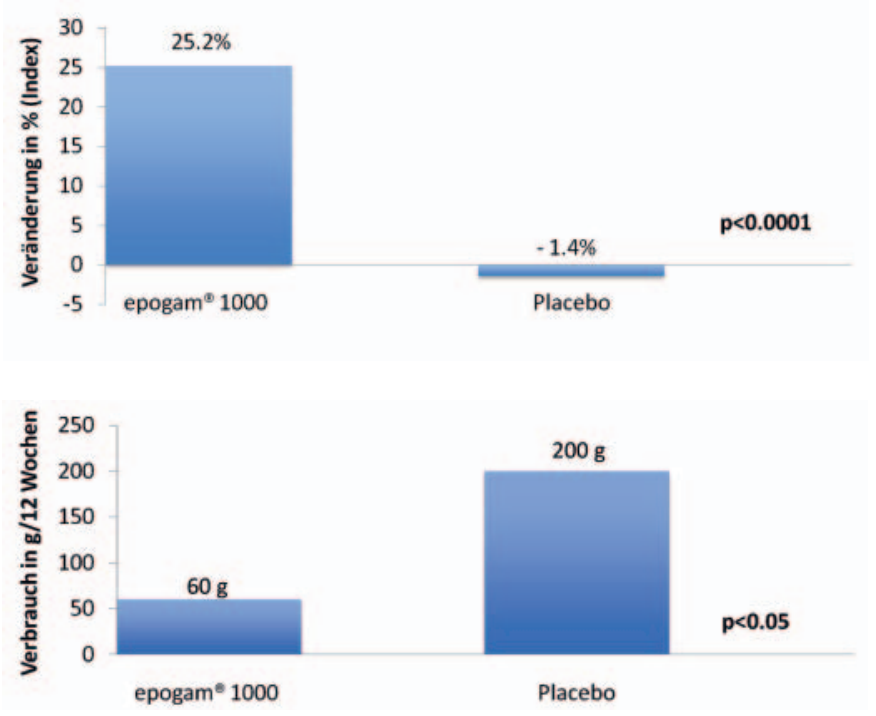

\section{Literatur}

1 Proksch E: Ungesättigte Fettsäuren; in Korting HC, Sterry W (Hrsg): Therapeutische Verfahren in der Dermatologie: Dermatika und Kosmetika. Berlin, Blackwell, 2001, pp 599-606.

2 Reinke C: Mehrfach ungesättigte Fettsäuren bei chronisch-entzündlichen Hautkrankheiten. Medicos 2003;4:9-10.

3 Stauber G: Bedeutung mehrfach ungesättigter Fettsäuren. Medicos 2010;11:20-22.

4 Horrobin DF: Essential fatty acid metabolism and its modification in atopic eczema. Am J Clin Nutr 2000;71:367-372.

5 Pirow N: Nachtkerzensamenöl bei atopischer Dermatitis. Aktuelle Medizin 2003;2:25-28.

6 Eichhorn J: Hochkonzentriertes Nachtkerzensamenöl bei entzündlichen Prozessen: In Studien geprüft, in der Praxis bewährt. Medical Tribune 2011; Nr. 01-02. 
7 Eichhorn J: Arachidonsäure - Wissenswertes und Hitlisten. www.ever.ch/ PDF/Hitliste_Arachidonsäure.pdf.

8 Mayr M, Eichhorn J: Gesunde Ernährung bei Rheuma. Stuttgart, Haug, 1999.

9 Arzneimittel-Kompendium der Schweiz ${ }^{\circledR}$, April 2009.

10 Pruthi S, et al: Vitamin E and evening primrose oil for management of cyclical mastalgia: a randomized pilot study. Altern Med Rev 2010;15:59_ 67.

11 Morse N, et al: A meta-analysis of randomized, placebo-controlled clinical trials of Efamol evening primrose oil in atopic eczema. Where do we go from here in light of more recent discoveries? Curr Pharm Biotechnol 2006;7:503-524.

12 Schalin M, et al: Evening primrose oil in the treatment of atopic eczema: effect on clinical status, plasma phospholipid fatty acids and circulation blood prostaglandins. Br J Dermatol 1987;117:11-19.

13 Morse PF, et al: Meta-analysis of placebo-controlled studies of the efficacy of epogam in the treatment of atopic eczema. Relationship between plasma essential fatty acid changes and clinical response. Br J Dermatol 1989;121: 75-90.

14 Tronnier $\mathrm{H}$, et al: Behandlungsstudie der Dermatitis atopica mit ungesättigten Fettsäuren. H+G 1993;68:562-572.

15 Martens-Loebenhoff J, et al: Pharmacokinetic data of gamma-linolenic acid on healthy volunteers after the administration of evening primrose oil (epogam). Int J Clin Pharmacol Ther 1998;36:363-366.

16 Jack AM, et al: Effects of diabetes and evening primrose oil treatment on responses of aorta, corpus cavernosum and mesenteric vasculature in rats. Life Sci 2002;71:1863-1877.

17 Teuscher E: Biogene Arzneistoffe. Fettsäuren und ihre Ester. Stuttgart, WVG, 1997, p 100.

18 Belch JJ, et al: Evening primrose oil in rheumatologic conditions. Am J Clin Nutr 2000;71(suppl 1):352-356.

19 Huntley AL, et al: Systematic review of herbal medicinal products for the treatment of menopausal symptoms. Menopause 2003;10:465-476.

20 Muggli R: Systemic evening primrose oil improves the biophysical skin parameter of healthy adults. Int J Cosmet Sci 2005;27:243-249.

21 Yoshimoto-Furuie K, et al: Effects of oral supplementation with evening primrose oil for six weeks on plasma essential fatty acids and uremic skin symptoms in hemodialysis patients. Nephron 1999;81:151-159.

22 AID Verbraucherdienst, 1998. 